\title{
Analysis of the shift in the superconducting transition under pressure in the Anderson-Hubbard two-orbital model
}

\author{
A. A. Kosov \\ Mari State University, Yoshkar-Ola, Mari El, 424001, Russia \\ E-mail: kosov@margu.mari.su
}

Recieved August 21, 1997

\begin{abstract}
The two-orbital Hubbard model is used to obtain formulas for the fermion excitation spectrum in the energy bands hybridized by Anderson's interaction. A transition to the Hubbard operators, which diagonalizes the one-site part of the Hamiltonian, allows us to use the Green's temperature function technique to take into account the interstitial jump term while studying the superconducting properties of the model. An analysis of the lower part of the energy spectrum leads to a formula for the superconducting transition temperature $T_{c}$ associated with the pairing of quasiparticles in one of the correlated bands. The dependence of $T_{c}$ on electron concentration and energy parameters determining the intraatomic correlation is studied. Proposing a simple relation between the value of pressure $(P)$ and width of the correlated band, the dependence of $T_{c}$ on the pressure was defined. Good agreement between the theoretical calculation of the dependence of $T_{c}$ on the pressure and the experimental results for $\mathrm{Y}_{1-x} \mathrm{Pr}_{x} \mathrm{Ba}_{2} \mathrm{Cu}_{3} \mathrm{O}_{7-\delta}$ is found. Comparison of the theoretical and experimental results for the dependence of $T_{c}$ and its derivative $d\left(\ln T_{c}\right) / d P$ on $\mathrm{Sr}$ and Bi-content $(x)$ in $\mathrm{La}_{2-x} \mathrm{Sr}_{x} \mathrm{CuO}_{4}$ has been made. It is concluded that the model under consideration can be used for the description of the shift in $T_{c}$ under pressure for a number of superconductors.
\end{abstract}

PACS: 74.20.-z

\section{Introduction}

The theoretical investigation of transition metal compounds is greatly complicated by the need to take into account not only the band effects but also the atom-like behavior of $d$ and $f$ states. Nevertheless, investigations of strongly correlated systems have increased during the last few years $[1,2]$. The enhanced interest in this problem is due to the conviction of many researches that strong electron correlations in the $d(f)$-electron subsystem ensure a comparatively high superconducting transition temperature $T_{c}$ in cuprate compounds like $\mathrm{La}_{2-x} \mathrm{Sr}_{x} \mathrm{CuO}_{4}, \mathrm{YBa}_{2} \mathrm{Cu}_{3} \mathrm{O}_{7-\delta}, \mathrm{Tl}_{2} \mathrm{Ba}_{2} \mathrm{CuO}_{y}$, and others. Investigation of the mechanisms of superconductivity of such systems is closely connected with the high-pressure investigation of these substances. One of the advantages of using high pressure technique is to change atomic distance without any substitution of components, which often causes some side effects $[3,4]$.
One of the popular models, which is used for the description of a strongly correlated system, is the Hubbard model [5]. Recently, Kosov and Shilov showed [6] that Hubbard's two-orbital degenerate model is quite promising for studying the superconducting transition in narrow-band materials. In contrast with Hubbard's classical single-orbit model [5], the two-orbit modification has a greater potential and can describe the behavior of two groups of strongly interacting electrons, i.e., conduction electrons and electrons localized at crystal lattice sites.

This work is devoted to the theoretical study of the superconducting transition and the high pressure effect in compounds, described by a unified Hamiltonian containing operators of Hubbard's two-orbital model and Anderson's interaction. Such unification considerably enhances the possibilities of Hubbard's two-orbital model and allows a more consistent analysis than, say, that in Refs. 7-9, of the interaction of nonlocalized and localized elec- 
trons by proceeding from the mixing of their oneparticle states. In order to develop the paper [6] we include here the Coulomb repulsive interaction of conduction electrons on the one site $U_{1}$. Taking into account the potential $U_{1}$ allows us to compare the results of the investigation of $T_{c}$, which were obtained here, with well-known results of others models.

In sec. 2 we derive the energy spectrum of a system described by the Hubbard-Anderson two-orbit model. In Secs. 3 and 4 we use the Green's temperature functions to study the energy spectrum in disperse bands. We obtained the operator responsible for superconducting pairing in the strongcoupling approximation and analyzed its energy dependence. On the basis of a numerical analysis we show that there exists an optimal range of variation of energy parameters, which corresponds to the maximum value of $T_{c}$. Special attention is focused on the dependence of $T_{c}$ on the potentials $U_{1}$ and $V_{0}$ (Anderson's hybridization) and on the singleparticle energy $E$. In Sec. 5 we calculate the effect of pressure on the superconducting transition temperature. A comparison of theoretical and experimental results for the dependence of $T_{c}$ on pressure in $\mathrm{Y}_{1-x} \mathrm{Pr}_{x} \mathrm{Ba}_{2} \mathrm{Cu}_{3} \mathrm{O}_{7-\delta}$, and for the dependence of $T_{c}$ and its derivative $d\left(\ln T_{c}\right) / d P$ on Sr- and Bi-content $x$ in $\mathrm{La}_{2-x} \mathrm{Sr}_{x} \mathrm{CuO}_{4}$ and $\mathrm{BaPb}_{1-x} \mathrm{Bi}_{x} \mathrm{O}_{3}$ has been made.

\section{Wave functions}

In order to describe the properties of the strongly correlated electron system we start from the Hamiltonian

$$
\begin{gathered}
\mathcal{H}=\mathcal{H}_{0}+\mathcal{H}_{\text {int }}=\sum_{i} \mathcal{H}_{0 i}+\sum_{i j s} t_{i j} C_{i s}^{+} C_{i s}, \quad \text { (1) } \\
\mathcal{H}_{0 i}=-\mu\left(n_{i a \uparrow}+n_{i a \downarrow}+n_{i c \uparrow}+n_{i c \downarrow}\right)+E\left(n_{i a \uparrow}+n_{i a \downarrow}\right)- \\
-H\left(n_{i a \uparrow}-n_{i a \downarrow}+n_{i c \uparrow}-n_{i c \downarrow}\right)+I n_{i a \uparrow} n_{i a \downarrow}+ \\
+U\left(n_{i a \uparrow}+n_{i a \downarrow}\right)\left(n_{i c \uparrow}+n_{i c \downarrow}\right)+U_{1} n_{i c \uparrow} n_{i c \downarrow}+ \\
+V_{0}\left(a_{i \uparrow}^{+} C_{i \uparrow}+a_{i \downarrow}^{+} C_{i \downarrow}+\text { H.c. }\right)
\end{gathered}
$$

where $C_{i s}^{+}, C_{i s}$ and $a_{i s}^{+}, a_{i s}$ are the field operators corresponding to free and localized electrons at the site $i$ with spin projection $s ; n_{\text {ias }}=a_{i s}^{+} a_{i s}$ and $n_{i c s}=C_{i s}^{+} C_{i s}$ are the operators of the electrons; $\mu$ is the chemical potential; $H$ is the applied magnetic field; $E$ is the one-particle energy of $a$-electrons; $I$, $U$, and $U_{1}$ are the energy parameters defining intraatomic correlation; $I$ is Hubbard interaction of localized electrons; $U$ is the interorbital Coulomb interaction of $c$ - and $a$-electrons; $U_{1}$ is the repulsive interaction of $c$-electrons at one site; $V_{0}$ is the matrix element responsible for hybridization of $c$ and $a$-electronic states (Anderson constant); and $\mathcal{H}_{\text {int }}$ describes the interstitial tunneling of $c$-electrons with transport integral $t_{i j}$.

Diagonalization of the single-cell Hamiltonian $\mathcal{H}_{0 i}$ and transition to the Hubbard operators $X_{p}^{q}$ lead to the following results for the wave function and the energy spectrum:

$$
\begin{gathered}
E_{0}=0, \Psi_{0}=|0,0\rangle-\text { vacuum state } \\
E_{A, B}=-\mu+(E-2 H) / 2 \pm\left(E^{2} / 4+V_{0}^{2}\right)^{1 / 2} ; \\
\Psi_{A, B}=\cos \alpha|+, 0\rangle \pm \sin \alpha|0,+\rangle ; \\
E_{C, D}=-\mu+(E+2 H) / 2 \pm\left(E^{2} / 4+V_{0}^{2}\right)^{1 / 2} ; \\
\Psi_{C, D}=\cos \alpha|-, 0\rangle \pm \sin \alpha|0,-\rangle ; \\
|s, 0\rangle=a_{i s}^{+}|0,0\rangle ;|0,+\rangle=C_{i s}^{+}|0,0\rangle ; \\
\cos \alpha=Z /\left(Z^{2}+V_{0}^{2}\right)^{1 / 2} ; \\
\sin \alpha=V_{0} /\left(Z^{2}+V_{0}^{2}\right)^{1 / 2} ;
\end{gathered}
$$

$$
Z= \begin{cases}\left(E^{2} / 4+V_{0}^{2}\right)^{1 / 2}+E / 2 & \text { if } E>0 \\ \left(E^{2} / 4+V_{0}^{2}\right)^{1 / 2}-E / 2 & \text { if } E<0\end{cases}
$$

It was mentioned in the introduction that the one-particle states $A, B, C$, and $D$ depend on the energy $V_{0}$ :

$$
\begin{gathered}
E_{F, G}=U-2 \mu+E \pm 2 H ; \\
\Psi_{F}=|+.+\rangle ; \quad \Psi_{G}=|-,-\rangle ; \\
E_{T}=U-2 \mu+E ; \\
\Psi_{s}=2^{-1 / 2}\{|+,-\rangle-|-,+\rangle\} ; \\
|+,+\rangle=a_{i \uparrow}^{+} C_{i \uparrow}^{+}|0,0\rangle ; \quad|-,-\rangle=a_{i \downarrow}^{+} C_{i \downarrow}^{+}|0,0\rangle ; \\
|+,-\rangle=a_{i \uparrow}^{+} C_{i \downarrow}^{+}|0,0\rangle .
\end{gathered}
$$

The energy of the remaining two-particle states, $E_{K}, E_{L}$, and $E_{M}$, can be obtained by using the cubic equation

$$
X^{3}+A X^{2}+B X+C=0
$$




$$
\begin{gathered}
A=-\left(I+U+U_{1}+3 E\right) ; \\
B=(I+2 E)(I+U)+U_{1}(3 E+U+I)-4 V_{0}^{2} ; \\
C=2 V_{0}^{2}\left(I+2 E+U_{1}\right)-U_{1}(E+U)(2 E+I) .
\end{gathered}
$$

The roots $X=\left\{X_{m}\right\}$ of Eq. (2) define the energy $E_{K, L, M} ; X_{m}=2 \mu+E_{m, m=K, L, M}$. The wave functions $\Psi_{K}, \Psi_{L}$, and $\Psi_{M}$, which correspond to the energy states $E_{K}, E_{L}$, and $E_{M}$, are presented in the Appendix. It must be emphasized that a consideration of two-particle states is the most significant aspect of this research, which distinguishes it from the widely discussed calculation of $T_{c}$ in the $t-J$ model [10], which does not directly take into consideration the two-particle electron states at a single site.

After exact diagonalization of the single-lattice part of the Hamiltonian (1), it can be represented in terms of Hubbard diagonal operators $X_{p}^{q}$ in the following form [6]:

$$
\begin{gathered}
\mathcal{H}_{0 i}=\sum_{P} E_{P} X_{P}^{P}, \\
P=0, A, B, \ldots, K, L, M .
\end{gathered}
$$

The operator $\mathcal{H}_{\text {int }}$ which is responsible for interstitial electron jumps can be represented as the quadratic form of nondiagonal Hubbard operators:

$$
\mathcal{H}_{\text {int }}=\sum_{i j s} t_{i j} g_{i s}^{*}(X) g_{j s}(X)
$$

The operators $C_{i s}^{+}$and $C_{i s}$ in $\mathcal{H}_{\text {int }}$ are expressed using the matrix defined in Eq. (A1) in terms of the Hubbard operators, and are given in terms of the operator functions $C_{i s}^{+}=g_{i s}^{*}(X)$ and $C_{i s}=g_{i s}(X)$, whose explicit form is given in the Appendix by Eqs. (A2) and (A3).

Since it is difficult to analyze operators (3) and (4) directly, we restrict our analysis to certain conditions for preliminary calculations. We assume that $I, U \gg U_{1},|E|,|\mu|$, and $V_{0}$. Also, we need to check our results according to two separate conditions: $E>0$ and $E<0$. For this reason, we use the intermediate symbols:

$$
\begin{aligned}
& Q= \begin{cases}B & \text { if } E>0 ; \\
A & \text { if } E<0 ;\end{cases} \\
& S= \begin{cases}D & \text { if } E>0 ; \\
C & \text { if } E<0\end{cases}
\end{aligned}
$$

This means, for example, that for the value $R_{K}^{S}$ we have

$$
R_{K}^{S}= \begin{cases}R_{K}^{D} & \text { if } E>0 \\ R_{K}^{C} & \text { if } E<0\end{cases}
$$

After the satisfaction of these conditions we need to consider the one-particle states $\Psi_{Q}$ and $\Psi_{S}$, the two-particle state $\Psi_{K}$, and the vacuum state $\Psi_{0}$ only. Furthermore, based on consideration of the low-lying part of the energy spectrum, we will obtain the formula for the superconducting transition temperature $T_{c}$ resulting from the paired quasiparticles in one of the correlated bands.

\section{The Green's functions}

According to Zyubin et al. [9] and Plakida et al. [10], who applied and developed the Green's function technique for solving such a problem, the above-mentioned energy levels of single-cell part of the Hamiltonian, correct wave functions, and familiar commutation relations for Hubbard operators can be used for finding the spectrum of elementary excitations of nonlocalized $c$-electrons in the strongcoupling approximation and for investigating their superconducting properties. Using these assumptions, we define the vector

$$
\Psi^{+}=\left(X_{Q}^{0}, X_{S}^{0}, X_{K}^{S}, X_{\bar{K}}^{Q}\right) .
$$

We then determine the temperature Green's functions [11]

$$
G\left(R_{m}, \tau ; R_{n}, \tau^{\prime}\right)=-\left\langle T_{\tau} \Psi\left(R_{m}, \tau\right) \Psi^{+}\left(R_{n}, \tau^{\prime}\right)\right\rangle
$$

The equations for the components of the Green's function $G$ are determined in the Appendix by Eq. (A4). We obtain these equations by assuming the existence of the order parameter $\Delta_{0}^{K}$ in the superconducting phase:

$$
\begin{gathered}
\Delta_{0}^{K}=\left\langle\Psi_{K}\left|X_{Q}^{0}\left(R_{m}\right) X_{\widetilde{K}}^{Q}\left(R_{m}\right)+X_{K}^{S}\left(R_{m}\right) X_{S}^{0}\left(R_{m}\right)\right| 0,0\right\rangle= \\
=\left\langle\Psi_{K}\left|X_{K}^{0}\left(R_{m}\right)\right| 0,0\right\rangle .
\end{gathered}
$$

The origin of the parameter $\Delta_{0}^{K}$ is closely related to the anomalous mean $\left\langle 2,0\left|C_{m \uparrow}^{+} C_{m \downarrow}^{+} \|\right| \mid 0,0\right\rangle$, which plays a fundamental role in the classical model of superconductivity of nonlocalized BCS electrons [12]. This can be verified, firstly, by using Eq. (A2) for the operators $C_{m \uparrow}^{+}$and $C_{m \downarrow}^{+} \downarrow$ and, secondly, through a detailed analysis of the symmetry properties of the state $\Psi_{K}$. It can be seen from Eq. (A1) that the wave function $\Psi_{K}$ is a linear combination of the initial functions $|0,2\rangle,|2,0\rangle$, $|T\rangle$ and, together with them, corresponds to the spin $S=0$ of the two groups of electrons, while the spin 
moments described by these states of the two particles are opposite. If $V_{0}=0$ (hybridization of localized and conduction electrons is absent), we have $\Psi_{K}=\Psi_{K}^{0}=|0,2\rangle$. It can be seen from definition (6) that $\Delta_{0}^{K}$ can be defined in terms of the anomalous functions $F_{Q K}^{0 Q}\left(p, \omega_{n}\right)$ and $F_{K S}^{S 0}\left(p, \omega_{n}\right)$ :

$$
\Delta_{0}^{K}=T \sum_{p} \sum_{n}\left[F_{Q K}^{0 Q}\left(p, \omega_{n}\right)+F_{K S}^{S 0}\left(p, \omega_{n}\right)\right] .
$$

The main determinant of Eqs. (A4) is

$$
\begin{gathered}
D=\left(\omega_{n}^{2}+\left(\xi_{10}\left(t_{p}\right)\right)^{2}\right)\left(\omega_{n}^{2}+\left(\xi_{20}\left(t_{p}\right)\right)^{2}\right)+ \\
+t_{p}^{2} \cos ^{2} \alpha\left(R_{K}^{S}\right)^{2}\left(\Delta_{0}^{K}\right)^{2}\left[\left(E_{K}+E_{S}-E_{Q}\right)^{2}+4 \omega_{n}^{2}\right] \\
\xi_{20,10}\left(t_{p}\right)=-\mu-\Delta / 2-\left[\cos ^{2} \alpha\left(n_{0}+n_{Q}\right)+\right. \\
\left.+\left(R_{K}^{S}\right)^{2}\left(n_{S}+n_{K}\right)\right] t_{p} / 2 \pm
\end{gathered}
$$

$\pm(1 / 2)\left\{\left[\cos ^{2} \alpha\left(n_{0}+n_{Q}\right)+\left(R_{K}^{S}\right)^{2}\left(n_{S}+n_{K}\right)\right] t_{p}+\Delta_{1}\right]^{2}+$ $\left.+4 \cos ^{2} \alpha\left(R_{K}^{S}\right)^{2}\left(n_{S}+n_{K}\right)\left(n_{0}+n_{Q}\right) t_{p}^{2}\right\}^{1 / 2}$,

$$
\begin{gathered}
\Delta=-\left(E_{K}+2 \mu\right) ; \\
\Delta_{1}=\Delta+E-\operatorname{sign}(E)\left[E^{2}+4 V_{0}^{2}\right]^{1 / 2} .
\end{gathered}
$$

The quantity $\xi_{20,10}$ is the energy spectrum of dispersed bands in the normal state, and $n_{0}, n_{O}$, $n_{S}$, and $n_{K}$ are the population densities of the energy states $\Psi_{0}, \Psi_{Q}, \Psi_{S}$, and $\Psi_{K}$, which satisfy the condition $\left(n_{S}+n_{K}\right)+\left(n_{0}+n_{Q}\right)=1$. For numerical calculations we will use one parameter for concentration $n$, where

$$
\left(n_{0}+n_{Q}\right)=1-n / 2 \text { and }\left(n_{K}+n_{S}\right)=n / 2 .
$$

The conditions (7c) are convenient for the halffilling of the $c$-band.

After solving the equation $D=0$, the following expression for the energy spectrum in the Anderson bands at $T<T_{c}$ can be found:

$$
\begin{gathered}
\xi_{2,1}^{2}=\left(\xi_{10}^{2}+\xi_{20}^{2}\right) / 2 \pm\left\{\left[\left(\xi_{10}^{2}-\xi_{20}^{2}\right) / 2\right]^{2}+\right. \\
+\cos ^{2} \alpha\left(R_{K}^{S}\right)^{2} t_{p}^{2}\left(\Delta_{0}^{K}\right)^{2}\left[2\left(\xi_{10}^{2}+\xi_{20}^{2}\right)-\left(E_{K}+E_{S}-\right.\right. \\
\left.\left.\left.-E_{Q}\right)^{2}+4 \cos ^{2} \alpha\left(R_{K}^{S}\right)^{2} t_{p}^{2}\left(\Delta_{0}^{K}\right)^{2}\right]\right\}^{1 / 2}
\end{gathered}
$$

Knowing the Green's function $G\left(p, \omega_{n}\right)$ and the electron concentrations for localized states with zero dispersion and using the completeness condi- tion (A5) for occupation numbers, we can determine the chemical potential $\mu$ for $\Delta_{0}^{K}=0$. For this purpose, we assume that the density of states in the dispersion region has a rectangular shape $[6,8,9]$ :

$$
\rho(\varepsilon)=\frac{1}{2 W} \theta\left(W^{2}-\varepsilon^{2}\right),
$$

where $2 W$ is the width of the $c$-band.

Carrying out calculations similar to those in Refs. 6 and 9, we obtain the expression for the chemical potential

$$
\begin{gathered}
\mu=-\frac{\Delta}{2}-\frac{W B_{+}}{2}+2\left(n_{K}+n_{S}\right) W\left(R_{K}^{S}\right)^{2}-\frac{P_{-}}{2} \\
P_{ \pm}=\left\{B_{+}^{2} W^{2} \pm 2 \Delta_{1} B_{-} W+\Delta_{1}^{2}\right\}^{1 / 2} \\
B_{ \pm}=\cos ^{2} \alpha\left(n_{0}+n_{Q}\right) \pm\left(R_{D}^{K}\right)^{2}\left(n_{K}+n_{S}\right)
\end{gathered}
$$

The chemical potential $\mu$ is determined by the concentration of electrons in the dispersed correlated band, which equals $n_{c}=\left(R_{K}^{S}\right)^{2}\left(n_{K}+n_{S}\right)$. The dependence of $n_{c}$ and $\mu$ on $V_{0}$ shows different behavior patterns in the cases $E>0$ and $E<0$. An increase of the hybridizing parameter $V_{0}$ leads to a decrease of $n_{c}$ when $E>0$ and to its increase when $E<0$.

\section{Temperature of the superconducting transition}

Before solving Eq. (7) for $T_{c}$, we must determine the Green's functions $F_{O K}^{0 Q}\left(p, \omega_{n}\right)$ and $F_{K S}^{S 0}\left(p, \omega_{n}\right)$ with the help of Eq. (A4). We write the following expression for these functions:

$$
F_{Q K}^{0 Q}\left(p, \omega_{n}\right)=
$$

$$
\begin{gathered}
=-t_{p} \Delta_{0}^{K}\left(R_{K}^{S}\right)^{2}\left(n_{K}+n_{S}\right)\left(i \omega_{n}-E_{Q}\right) \frac{i \omega_{n}-E_{Q}+E_{K}-B_{-} t_{p}}{\Omega_{0}} ; \\
F_{K S}^{S 0}\left(p, \omega_{n}\right)=
\end{gathered}
$$

$$
\begin{gathered}
=-t_{p} \Delta_{0}^{K} \cos ^{2} \alpha\left(n_{0}+n_{Q}\right)\left(i \omega_{n}+E_{S}-E_{K}\right) \frac{i \omega_{n}+E_{S}+B_{-} t_{p}}{\Omega_{0}} \\
\Omega_{0}=\left(\xi_{2}^{2}+\omega_{n}^{2}\right)\left(\xi_{1}^{2}+\omega_{n}^{2}\right)
\end{gathered}
$$

where $\xi_{2}$ and $\xi_{1}$ are given in Eq. (7b) 
Taking the relation (7) into account and considering that $\Delta_{0}^{K}\left(T_{c}\right)=0$, we obtain the equation for the critical temperature $T_{c}$

$$
\begin{gathered}
1=\int_{-W}^{W} \frac{t J(t) \tanh \left[\xi_{10}(t) / 2 T_{c}\right] d t}{4 W\left[\xi_{20}^{2}(t)-\xi_{10}^{2}(t)\right] \xi_{10}(t)} ; \\
J(t)=\cos ^{2}(\alpha)\left(n_{0}+n_{Q}\right)\left(\xi_{10}(t)+\right. \\
\left.+E_{S}-E_{K}\right)\left(\xi_{10}(t)+E_{S}+B_{-} t_{p}\right)+ \\
+\left(R_{K}^{S}\right)^{2}\left(n_{K}+n_{S}\right)\left(\xi_{10}(t)-E_{Q}\right)\left(\xi_{10}(t)-\right. \\
\left.-E_{Q}+E_{K}-B_{-} t_{p}\right) ;
\end{gathered}
$$

here $\xi_{10}(t)$ and $\xi_{20}(t)$ are determined by Eq. (7a).

In order to solve Eq. (10) approximately, we determine the value of $t_{0}$ from the equation $\xi_{10}\left(t_{0}\right)=0\left(-W<t_{0}<W\right)$ :

$$
t_{0}=\frac{(2 \mu+\Delta)^{2}-\Delta_{1}^{2}}{2\left[\Delta_{1} B_{-}+B_{+}(2 \mu+\Delta)\right]} .
$$

The approximate formula for $T_{c}$ following from Eq. (10) and $\xi_{10}\left(t_{0}\right)=0$ has the form

$$
\begin{gathered}
\frac{T_{c}}{2 W}=0.57\left[-\frac{\xi_{10}(-W) \xi_{10}(W)}{W^{2}}\right]^{1 / 2} \exp \left[-1 / A\left(n, t_{0}\right)\right] ; \\
\xi_{10}(-W)=-2\left(R_{K}^{S}\right)^{2}\left(n_{K}+n_{S}\right) W \\
\xi_{10}(W)=-\mu+\frac{B_{+} W-P_{+}-\Delta}{2} ; \\
A\left(n, t_{0}\right)=\Gamma\left(n, t_{0}\right) / \Lambda\left(n, t_{0}\right) ; \\
\Gamma\left(n, t_{0}\right)=t_{0}\left[\cos ^{2} \alpha\left(n_{0}+n_{Q}\right)\left(E_{S}-E_{K}\right)\left(E_{S}+B_{-} t_{0}\right)+\right. \\
\left.+\left(R_{K}^{S}\right)^{2}\left(n_{K}+n_{S}\right) E_{Q}\left(E_{Q}-E_{K}+B_{-} t_{0}\right)\right] ;
\end{gathered}
$$

$\Lambda\left(n, t_{0}\right)=W\left[B_{+}(2 \mu+\Delta)+B_{-} \Delta_{1}\right]\left(2 \mu+\Delta-B_{+} t_{0}\right)$.

The quantity $A\left(n, t_{0}\right)$ plays the role of the quasiparticle scattering amplitude with different spin orientations. The attraction between quasiparticles in a correlated band takes place under the conditions

$$
A\left(n, t_{0}\right)>0 ; \quad-W<t_{0}<W ; \quad \xi_{10}(W) \geq 0 .
$$

The conditions (12) can be used to determine the concentrations $n_{S 1}<n<n_{S 2}$, for which $T_{c} \neq 0$. Solving the equation $\xi_{10}(W)=0$ gives the following value for $n_{S 2}$ :

$$
n_{S 2}=2 \cos ^{2} \alpha /\left[\cos ^{2} \alpha+\left(R_{K}^{S}\right)^{2}\right] .
$$

The condition $A\left(n, t_{0}\right)=0$ gives the following result for $n_{S 1}$ :

$$
n_{S 1}=\frac{2 \cos ^{2} \alpha}{\cos ^{2} \alpha+2\left(R_{K}^{S}\right)^{2}} .
$$

In particular, if we set $V_{0}=0$ and $E>0$ in (12a) and (12b), we obtain $n_{S 1}=2 / 3$ and $n_{S 2}=1$. This result has been obtained in Ref. 7 in the frame of the one-orbit Hubbard model (the so-called «kinematics mechanism of superconductivity»).

\section{Pressure effects}

Many investigations of high- $T_{c}$ superconductors have been performed at high pressure $[3,4,13,14]$. The application of pressure usually strongly affects the crystal structure of materials. High-pressure investigations of elastic properties often give important information about the relation between $T_{c}$ and crystal symmetry. Applying the two-orbit Anderson-Hubbard model to describe the pressure dependence of $T_{c}$, we chose to study the energy parameters, which more sensitively depend on the pressure $P$. Since $U, I, U_{1}, V_{0}$, and $E$ are on-site properties, their pressure dependence can be ignored. The transport integral $t_{p}$ depends on the spatial distribution of atoms and can be changed by the applied pressure. Let us consider the region where $W$ depends linearly on $t_{p}\left(W \sim t_{p}\right)$. According to Ref. 15, the transport integral in metal oxides can be expressed in terms of the lattice parameters of the $\mathrm{CuO}_{2}$ planes $a, b$, and $c$ by the formulas

$$
t_{\|}=h^{2} /\left(2 m_{\|} a^{2}\right) ; \quad t_{\perp}=h^{2} /\left(2 m_{\perp} c^{2}\right),
$$

where $m_{\|}$and $m_{\perp}$ are the respective effective masses.

Disregarding pressure dependence of the effective masses, we estimate the magnitude of $d W / d P$ :

$$
d W / d P \sim d t_{p} / d P \sim-2 W(d \ln a / d P)=2 W k_{a} .
$$

Here $k_{a}, k_{b}$, and $k_{c}$ are the compressibility components along each crystallographic direction as defined by 


$$
\begin{gathered}
k_{a}=-d \ln a / d P ; \quad k_{b}=-d \ln b / d P ; \\
\text { and } k_{c}=-d \ln c / d P .
\end{gathered}
$$

In order to simplify the numerical estimation of these quantities, we consider the case $k_{a}=k_{b}=k_{c}$. Using the relations (13), we obtain the expression for the dependence of the band width on the pressure $P$

$$
W(P)=W(P=0) \exp \left(2 k_{a} P\right) .
$$

Equations (11) and (14) allow us to express $T_{c}$ as a function of pressure. Using the expression (13), it is possible to obtain equations for $d T_{c} / d P$ and $d \ln T_{c} d P$ :

$$
d T_{c} / d P=T_{c}\left(d \ln T_{c} / d P\right) \sim 2 W k_{a}\left(d T_{c} / d W\right) ;
$$

$$
\begin{gathered}
\frac{d T_{c}}{d W}=\frac{T_{c}}{2 W}\left\{1+2 W\left[B_{-}+\left(B_{+}^{2}\left(P_{+}-P_{-}\right) W-\right.\right.\right. \\
\left.\left.\left.-\Delta_{1} B_{-}\left(P_{+}+P_{-}\right)\right) / 2 P_{+} P_{-}\right]+\frac{2 W}{A^{2}\left(n, t_{0}\right)} \frac{d A\left(n, t_{0}\right)}{d W}\right\} \\
\frac{d A\left(n, t_{0}\right)}{d W}=-\frac{A\left(n, t_{0}\right)}{W}+ \\
+\Lambda\left(n, t_{0}\right)^{-1}\left\{\frac{2(2 \mu+\Delta)-2 B_{+} t_{0}}{B_{+}(2 \mu+\Delta)+\Delta_{1} B_{-}} \times\right. \\
+\left(\cos ^{2} \alpha\left(n_{0}+n_{Q}\right)\left(E_{S}-E_{K}\right)\left(E_{S}+2 B_{-} t_{0}\right)+\right. \\
+A\left(n, t_{0}\right) B_{+}\left(n_{K}+n_{S}\right) E_{Q}\left(E_{Q}-E_{K}+2 B_{-} t_{0}\right)+ \\
\left.\frac{d \mu}{d W}=2\left(B_{+}(2 \mu+\Delta)+\Delta_{1} B_{-}\right)\right]- \\
\left.-2 W A\left(n, t_{0}\right)\left[2 B_{+}(2 \mu+\Delta)+\Delta_{1} B_{-}-B_{+}^{2} t_{0}\right]\right\} \frac{d \mu}{d W} \\
\left.n_{K}+n_{S}\right)-\frac{B_{+}}{2}-\frac{W B_{+}^{2}-\Delta_{1} B_{-}}{2 P_{-}}
\end{gathered}
$$

\section{Discussion}

\section{Superconducting transition}

Expression (11) has the standard form of the BCS theory. It should be noted that the amplitude $A\left(n, t_{0}\right)$ directly depends on the values $\cos ^{2} \alpha$ and
$\left(R_{K}^{S}\right)^{2}$ [see (A3)], which determine, according to (A3), the efficiency of the transition between states $\Psi_{Q}, \Psi_{S}, \Psi_{K}$, and $\Psi_{0}$. As was mentioned above, in the cases $V_{0}=0$ (hybridization of $a$ and $c$ electrons is absent) and $E>0$, Eq. (11) describes the results of the well-known kinematics mechanism of superconductivity [7]. If we insert $U_{1}=0$ into (11), we obtain the results of Kosov and Shilov [6]. In order to simplify the analysis of the dependence of $T_{c}$ on the parameters of the problem, we carried out detailed numerical calculations on the basis of Eq. (11). The results are shown in Figs. 1, $a-f$. As expected, the concentration dependence of $T_{c} / 2 \mathrm{~W}$ shown in Figs. 1, $a-f$ is bell-shaped with two critical concentrations $n_{S 1}$ and $n_{S 2} \quad\left(T_{c} \neq 0\right.$ for $n_{S 1}<n<n_{S 2}$ ). As shown in Fig. 1, $a$, an increase in $V_{0}$ when $E>0$ leads to a decrease of the superconducting transition temperature and to a decrease of the concentrations $n_{S 1}$ and $n_{S 2}$. In this case Anderson's hybridization partly localizes the electrons and decreases the concentration of carriers in the correlated band $n_{c}=\left(R_{K}^{S}\right)^{2}\left(n_{K}+n_{S}\right)$. It contributes to the decrease of $T_{c}$ and shifts the curve $T_{c}=T_{c}\left(n_{c}\right)$ to the region of small concentration. If $E<0$, Anderson's hybridization partly delocalizes electrons and increases the concentration of carriers in the lower correlated band $n_{c}=\left(R_{K}^{S}\right)^{2}\left(n_{K}+n_{S}\right)$. In this case (Fig. 1,b), the maximum of the curve $T_{c}=T_{c}\left(n_{c}\right)$ increases sharply with increasing value of $V_{0} / W$. Such behavior of $T_{c}\left(n_{c}\right)$ is just the opposite when $E>0$.

The dependence of $T_{c}\left(n_{c}\right)$ on $E / W(E>0$ in Fig. $1, c$ and $E<0$ in Fig. 1,d can be seen in Fig. 1,c,d. Figure1,e,f shows the dependence of $T_{c}$ on $U_{1}$ in different cases, $E>0(a)$ and $E<0(b)$.

\section{Pressure Effects}

To study the capability of the model presented here to describe the pressure effect on $T_{c}$, we attempted to explain the results of a high pressure study of $\mathrm{Y}_{1-x} \mathrm{Pr}_{x} \mathrm{Ba}_{2} \mathrm{Cu}_{3} \mathrm{O}_{7-y}$ by Neumeier et al. [14] According to Ref. 16, the substitution of $\mathrm{Pr}^{4+}$ for $\mathrm{Y}$ decreases the number of in-plane holes in $\mathrm{Y}_{1-x} \mathrm{Pr}_{x} \mathrm{Ba}_{2} \mathrm{Cu}_{3} \mathrm{O}_{7-y}$. In order to take into account this argument we therefore must change the population densities in Eq. (7c):

$$
\left(n_{K}+n_{S}\right)=n / 2 \rightarrow\left(2\left(R_{K}^{S}\right)^{2}\right)\left(n_{c}-x_{P}\right)
$$

were $x_{P}$ is the concentration of $\operatorname{Pr}^{4+}$ ions.

Using Eqs. (7c), (11), (14), and (15), we obtain an expression which allows us to find the dependence of $T_{c}=T_{c}\left(P, x_{P}\right)$. Initial compressibility data 

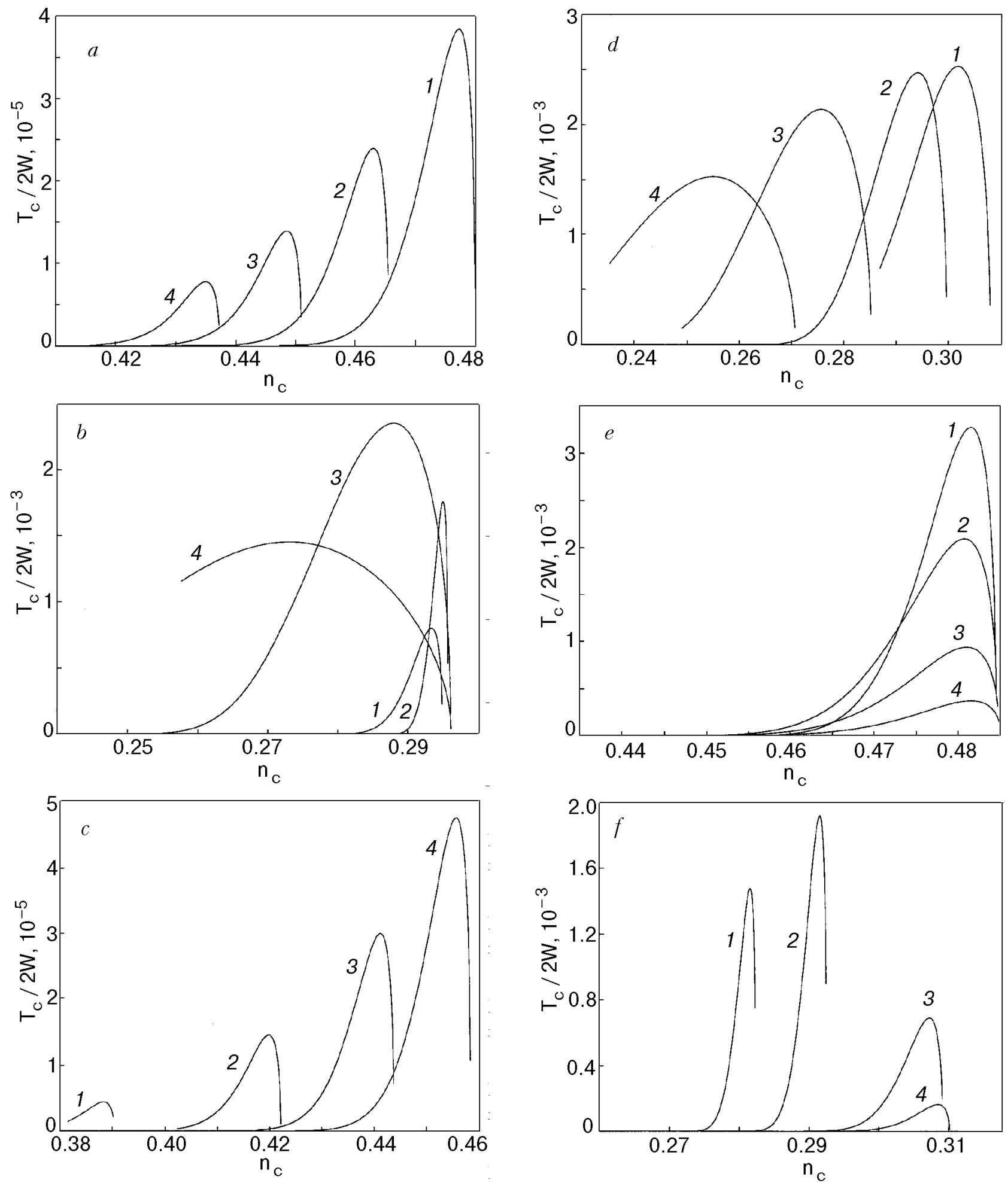

Fig. 1. Dependence of the function $T_{c} / 2 W$ on the carriers concentration $n_{c}$ for the following values of the parameters: $E / W=2.0, U / W=6.0, U_{1} / W=3.0, I / W=10.0$ at different values of $V_{0} / W: 0.5$ (1), 0.7 (2), 0.9 (3), 1.1 (4) (a); $E / W=-3.0, U / W=6.0, U_{1} / W=4.0, I / W=10.0$ at different values of $V_{0} / W: 1.0(1), 1.2(2), 1.4$ (3), 1.5 (4) (b); $V_{0} / W=1.0, U / W=6.0, U_{1} / W=2.5, I / W=10.0$ at different values of $E / W: 1.0(1), 1.5$ (2), 2.0 (3), 2.5 (4) (c); $V_{0} / W=1.2, U / W=6.0, U_{1} / W=2.0, I / W=8.0$ at different values of $E / W:-1.5(1),-2.0(2),-2.5(3),-2.8(4)(d)$; $E / W=1.0, V_{0} / W=0.2, U / W=6.0, I / W=10.0$ at different values of $U_{1} / W: 0.5$ (1), 1.0 (2), 1.5 (3), 2.0 (4) (e); $E / W=-1.5, V_{0} / W=1.0, U / W=6.0, I / W=8.0$ at different values of $U_{1} / W: 1.5(1), 2.0(2), 3.0(3), 4.0(4)(f)$. 


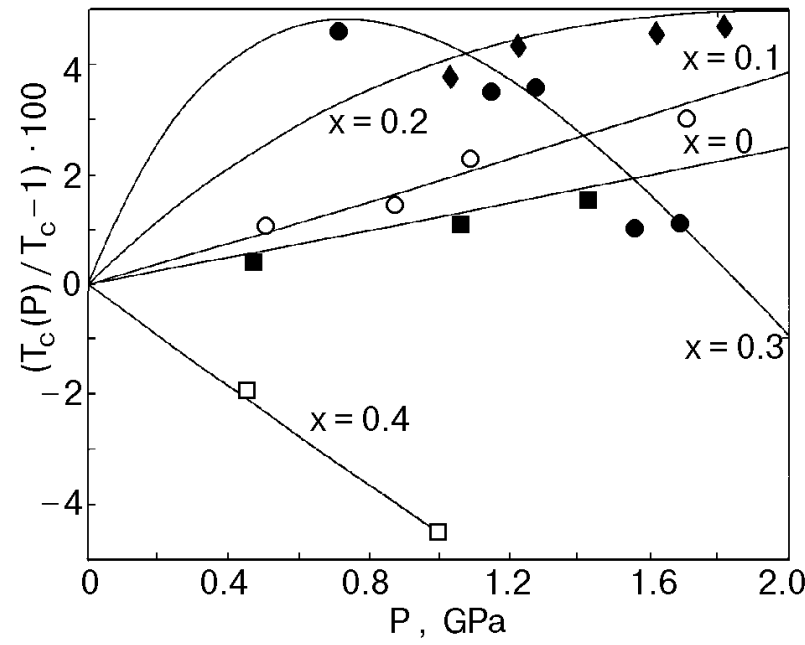

Fig. 2. Experimental data from Ref. 14 and theoretical curves for the percentage shift in the superconducting transition temperature $T_{c}$ under pressure for $\mathrm{Y}_{1-x} \mathrm{Pr}_{x} \mathrm{Ba}_{2} \mathrm{Cu}_{3} \mathrm{O}_{7-y}$ compounds. The solid lines show the results of numerical calculations using Eq. (15) for the following values of the parameters: $E / W=-2.0, \quad V_{0} / W=1.4, \quad U / W=3.0, \quad I / W=8.0$, $U_{1} / W=2.0, n_{c}=x, k_{a}=2.4 \cdot 10^{-3} \mathrm{GPa}^{-1}$.

for polycrystalline $\mathrm{YBa}_{2} \mathrm{Cu}_{3} \mathrm{O}_{6.93}$ and $\mathrm{YBa}_{2} \mathrm{Cu}_{3} \mathrm{O}_{6.6}$ of orthorhombic symmetry were obtained by using a hydrostatic He-gas pressure cell in a neutron diffractometer. According to Ref. 17, the values of $k_{a}$ and $k_{b}$ for these compounds are respectively $\quad k_{a}=2.22 \cdot 10^{-3} \mathrm{GPa}^{-1}, \quad 2.40 \cdot 10^{-3} \mathrm{GPa}^{-1}$, $k_{b}=1.65 \cdot 10^{-3} \mathrm{GPa}^{-1}$, and $2.14 \cdot 10^{-3} \mathrm{GPa}^{-1}$. In the present numerical calculations we used the value $k_{a}=k_{b}=2.42 \cdot 10^{-3} \mathrm{GPa}^{-1}$. Figure 2 shows the dependence of $T_{c}=T_{c}\left(P, x_{P}\right)$ and the results of a high pressure study by Neumeier at al. [14] on $\mathrm{Y}_{1-x} \mathrm{Pr}_{x} \mathrm{Ba}_{2} \mathrm{Cu}_{3} \mathrm{O}_{7-y}$ for a different Pr concentrations. We found that the best agreement between this model and experimental data takes place when the intraatomic parameters of energies are $U=$ $=3 \mathrm{eV}, U_{1}=2 \mathrm{eV}, I=8 \mathrm{eV}, E=-2 \mathrm{eV}, V_{0}=$ $=1.4 \mathrm{eV}$, and $W(P=0)=1 \mathrm{eV}$. These values are typical of metal oxides [18]. In Figs. 3 and 4 we compare the theoretical and experimental results [19-21] for the dependence of $T_{c}$ and its derivative $d\left(\ln T_{c}\right) / d P$ [by using expression (15)] on $\mathrm{Sr}$ and Bi-content $x$ in $\mathrm{La}_{2-x} \mathrm{Sr}_{x} \mathrm{CuO}_{4}$ and in $\mathrm{BaPb}_{1-x} \mathrm{Bi}_{x} \mathrm{O}_{3}$. An analysis of the dependence of $d\left(\ln T_{c}\right) / d P$ on the concentration of carriers $n_{c}$ based on the results presented in Figs. 3 and 4, indicates that an increase in the band width $W$ leads to a change of sign of the derivative $d\left(\ln T_{c}\right) / d P$ from positive at small values of $W$ (Fig. $3, b$ ) to negative at large values of $W$ (Fig. $4, b$ ). The results shown in Figs. 3 and 4 are
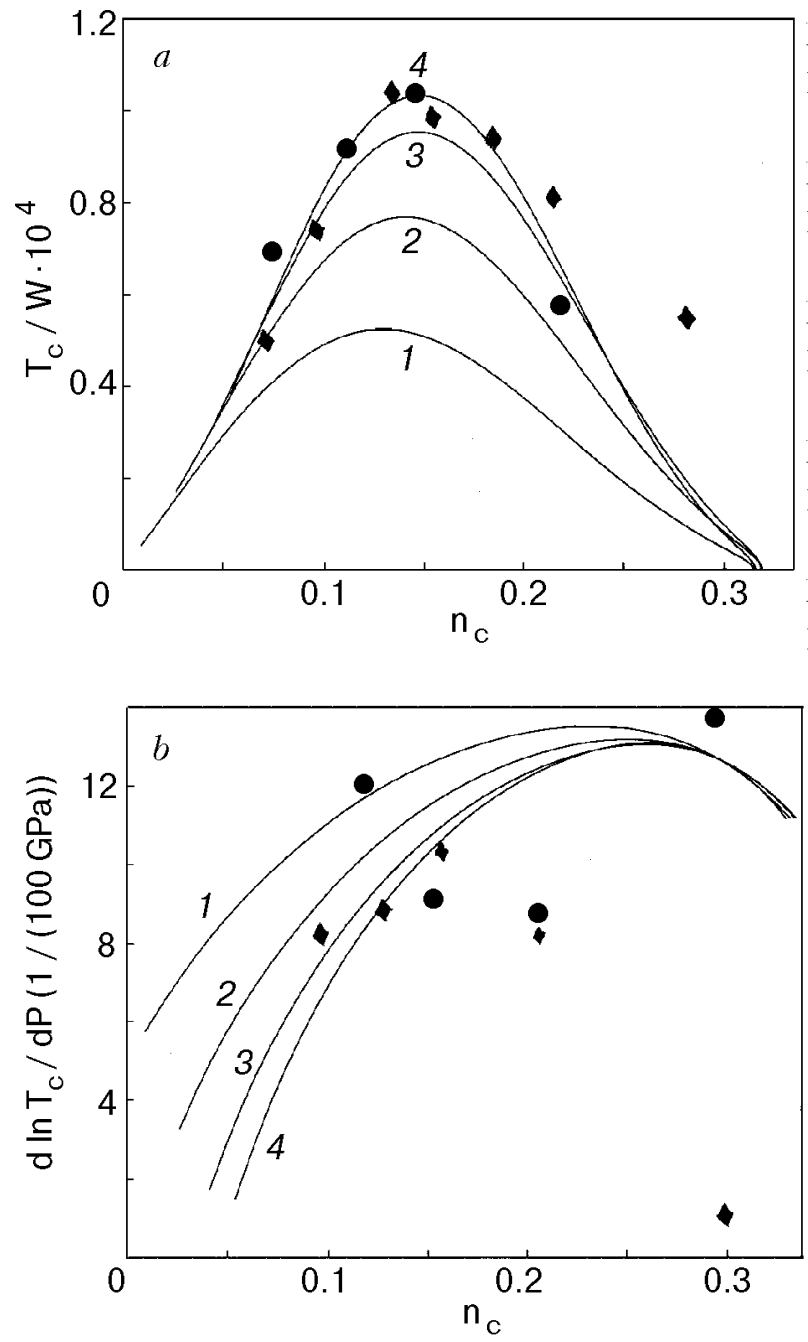

Fig. 3. Experimental data and theoretical curves for the dependence of $T_{c}(a)$ and its derivative $d\left(\ln T_{c}\right) / d P(b)$ on $\mathrm{Sr}$ content $x$ in $\mathrm{La}_{2-x} \mathrm{Sr}_{x} \mathrm{CuO}_{4}$ (electron concentration $n$ ) ( $\bullet$ Ref. 19; - Ref. 20). Solid lines show the results of numerical calculations using Eq. (15) for the following values of the parameters: $\quad V_{0} / W=1.25, \quad U / W=3.0, \quad I / W=5.0$, $U_{1} / W=1.0, n_{c}=x, k_{a}=8.0 \cdot 10^{-3} \mathrm{GPa}^{-1}, P=2 \mathrm{GPa}$ at different $E / W$ : -0.5 (1), -0.75 (2), -1.0 (3), -1.25 (4).

basically in accordance with the experimental results regarding the pressure dependence of the superconducting transition of many metal oxides. The dependence of $T_{c}$ on the width $W$ of the correlated band, shown in Fig. 5, also indicates that the Anderson-Hubbard two-orbital model, in principle, is able to describe the pressure dependence of $T_{c}$ for various high-temperature superconductors [22] $\left(\mathrm{Tl}_{2} \mathrm{Ba}_{2} \mathrm{CaCu}_{2} \mathrm{O}_{8}, \mathrm{YBa}_{2} \mathrm{Cu}_{4} \mathrm{O}_{8}\right.$, and others).

This research has been done under the auspices of the Junior Faculty Development Program and was supported by the U.S. Information Agency and Office of Sponsored Programs and Research of Bowling Green State University (BGSU), Ohio. 

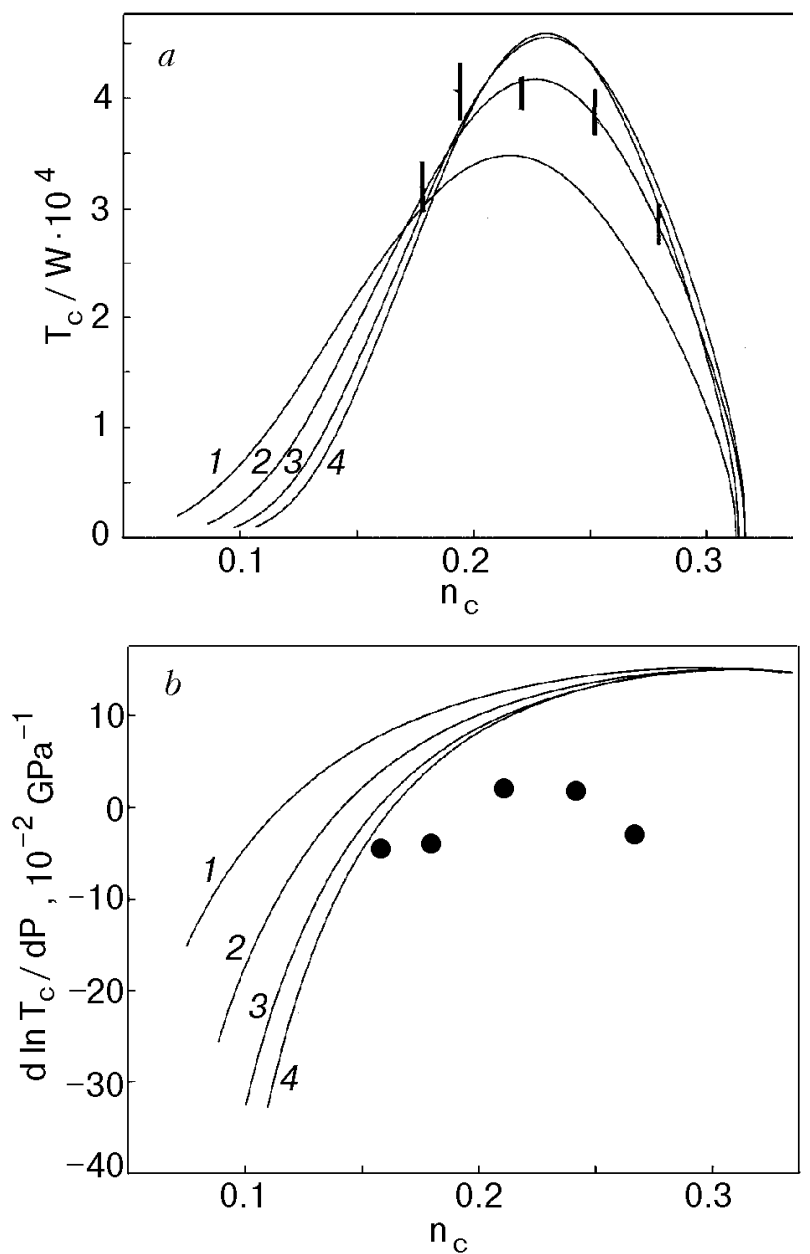

Fig. 4. Experimental data and theoretical curves for the dependence of $T_{c}(a)$ and its derivative $d\left(\ln T_{c}\right) / d P(b)$ on $\mathrm{Bi}$ content $x$ in $\mathrm{BaPb}_{1-x} \mathrm{Bi}_{x} \mathrm{O}_{3}$ (electron concentration $n$ ) $(\bullet$ Ref. 21). Solid lines show the results of numerical calculations using Eq. (15) for the following values of the parameters: $V_{0} / W=1.0, \quad U / W=2.4, I / W=4.0, \quad U_{1} / W=0.8, \quad n_{c}=x$, $k_{a}=2.29 \cdot 10^{-3} \mathrm{GPa}^{-1}, P=2 \mathrm{GPa}$ at different $E / W:-0.4(1)$, -0.6 (2), -0.8 (3), -1.0 (4).

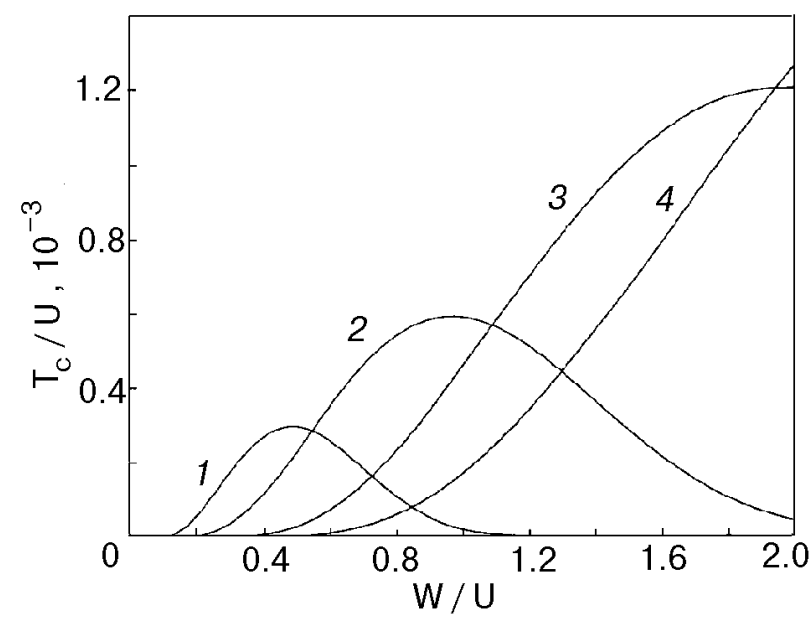

Fig. 5. Dependence of the function $T_{d} / 2 W$ on the width of the band for the following values of the parameters: $E / W=-1.5$, $V_{0} / W=1.0, \quad U / W=6.0, \quad I / W=8.0$ at different $U_{1} / W$ : $1.5(1), 2.0(2), 3.0(3), 4.0(4)$.
I am grateful to Ms. C. Crow, Dr. A. M. Boccanfuso, and Dr. D. D. Gehring for their encouragement to carry out scientific and educational research at BGSU. I thank Dr. R. Boughton for interesting discussions.

\section{Appendix}

1. The wave functions of the two-particle states $K, L, M$ can be determined by the standard procedure after calculating the roots of the cubic Eq. (3) :

$$
\begin{aligned}
& \left(\begin{array}{l}
\Psi_{L} \\
\Psi_{M} \\
\Psi_{K}
\end{array}\right)=\left(\begin{array}{lll}
A_{1} & A_{2} & A_{3} \\
B_{1} & B_{2} & B_{3} \\
C_{1} & C_{2} & C_{3}
\end{array}\right)\left(\begin{array}{l}
|2,0\rangle \\
|T\rangle \\
|0,2\rangle
\end{array}\right), \\
& A_{1}=\left(1+A_{21}^{2}+A_{31}^{2}\right)^{-1 / 2} ; \quad A_{2}=A_{21} A_{1} ; \\
& A_{3}=A_{31} A_{1} \text {; } \\
& A_{21}=\left(E_{L}-2 E-I\right)^{2} / 2 V_{0}^{2} \text {; } \\
& A_{31}=\left(E_{L}-2 E-I\right)^{2} /\left(E_{L}-U_{1}\right)^{2} \\
& B_{2}=\left(1+B_{12}^{2}+B_{32}^{2}\right)^{-1 / 2} ; B_{1}=B_{12} B_{2} ; \\
& B_{3}=B_{32} B_{2} \\
& B_{12}=\frac{\left(E_{M}+2 E-U\right)\left(E_{M}-U_{1}\right)-2 V_{0}^{2}}{\sqrt{2} V_{0}\left(E_{M}-U_{1}\right)} ; \\
& B_{32}=\sqrt{2} V_{0} /\left(E_{M}-U_{1}\right) \text {; } \\
& C_{3}=\left(1+C_{13}^{2}+C_{23}^{2}\right)^{-1 / 2} ; \quad C_{1}=C_{13} C_{3} ; \\
& C_{2}=C_{23} C_{3} \\
& C_{13}=\left[\left(E_{K}-E-U\right)\left(E_{K}-U_{1}\right)-2 V_{0}^{2}\right] /\left[2 V_{0}^{2}\right] ; \\
& C_{23}=\left(E_{K}-U_{1}\right) / \sqrt{2} V_{0} ; \\
& |T\rangle=2^{-1 / 2}\{|+,-\rangle+|-,+\rangle\} ; \quad|2,0\rangle=a_{i \uparrow}^{+} a_{i \downarrow}^{+}|0,0\rangle ; \\
& |0,2\rangle=C_{i \uparrow}^{+} C_{i \downarrow}^{+}|0,0\rangle . \\
& |T\rangle=2-1 / 2||+,-\rangle+|-,+\rangle\} ;|2,0\rangle=a_{i \uparrow}^{+} a_{i \downarrow}^{+}|0,0\rangle
\end{aligned}
$$

2. Having determined the reciprocal matrix $\left\{a_{i j}\right\}^{-1}$ [the matrix $\left\{a_{i j}\right\}$ was written in Eq. (A 1)] and using the expressions for the functions $\Psi_{Q, S}$, we can express the field operators $C_{\text {is }}^{+}$in terms of Hubbard's operators: 


\section{a. $E>0$;}

$$
\begin{gathered}
C_{i \uparrow}^{+}=\cos \alpha X_{B}^{0}+R_{D}^{K} X_{K}^{D}=g_{i \uparrow}^{*}(X) ; \\
C_{i \downarrow}^{+}=\cos \alpha X_{D}^{0}+R_{B}^{K} X_{K}^{B}=g_{i \downarrow}^{*}(X) ; \\
R_{D}^{K}==R_{B}^{K}=
\end{gathered}
$$

$=\cos \alpha\left(A_{1} B_{2}-A_{2} B_{1}\right)+\sin \alpha\left(A_{1} B_{3}-A_{3} B_{1}\right) / \sqrt{2}$.

b. $E<0$;

$$
\begin{gathered}
C_{i \uparrow}^{+}=\cos \alpha X_{A}^{0}+R_{C}^{K} X_{K}^{D}=g_{i \uparrow}^{*}(X) ; \\
C_{i \downarrow}^{+}=\cos \alpha X_{C}^{0}+R_{A}^{K} X_{K}^{B}=g_{i \downarrow}^{*}(X) ; \\
R_{C}^{K}=R_{A}^{K}= \\
=\cos \alpha\left(C_{1} A_{3}-A_{1} C_{3}\right) / \sqrt{2}+\sin \alpha\left(C_{1} A_{2}-C_{2} A_{1}\right) .
\end{gathered}
$$

3. After writing the equation of motion for function (6) using the Hamiltonian expressed by Eqs. (3) and (4), we obtain the following expressions for the components of the Green's function $\left[G_{q s}^{p r}\left(\tau, \tau^{\prime}\right)=-\left\langle T_{\tau} X_{q}^{p}(\tau) X_{s}^{r}\left(\tau^{\prime}\right)\right\rangle=-F_{q s}^{p r}\left(\tau, \tau^{\prime}\right)\right]$ in the momentum-frequency representation:

$$
\left(\begin{array}{c}
n_{d}+n_{k} \\
0 \\
0 \\
0
\end{array}\right)=\left(\begin{array}{llll}
b_{11} & b_{12} & b_{13} & b_{14} \\
b_{21} & b_{22} & b_{23} & b_{24} \\
b_{31} & b_{32} & b_{33} & b_{34} \\
b_{41} & b_{42} & b_{43} & b_{44}
\end{array}\right)\left(\begin{array}{c}
G_{D K}^{K D}\left(p, \omega_{n}\right) \\
G_{O K}^{B D}\left(p, \omega_{n}\right) \\
F_{K K}^{B D}\left(p, \omega_{n}\right) \\
F_{D K}^{O D}\left(p, \omega_{n}\right)
\end{array}\right)
$$

(A4)

$$
\begin{gathered}
\left(\begin{array}{c}
n_{b}+n_{0} \\
0 \\
0 \\
0
\end{array}\right)=\left(\begin{array}{llll}
c_{11} & c_{12} & c_{13} & c_{14} \\
c_{21} & c_{22} & c_{23} & c_{24} \\
c_{31} & c_{32} & c_{33} & c_{34} \\
c_{41} & c_{42} & c_{43} & c_{44}
\end{array}\right)\left(\begin{array}{c}
G_{O B}^{B O}\left(p, \omega_{n}\right) \\
G_{D B}^{K O}\left(p, \omega_{n}\right) \\
F_{K B}^{B O}\left(p, \omega_{n}\right) \\
F_{D B}^{O O}\left(p, \omega_{n}\right)
\end{array}\right) \\
b_{11}=i \omega_{n}+E_{D}-E_{K}-\left(R_{D}^{K}\right)^{2}\left(n_{d}+n_{k}\right) t_{p} \\
b_{22}=i \omega_{n}-E_{B}-\cos ^{2} \alpha\left(n_{0}+n_{b}\right) t_{p} \\
b_{33}=i \omega_{n}-E_{B}+E_{K}+\left(R_{D}^{K}\right)^{2}\left(n_{d}+n_{k}\right) t_{p} \\
b_{44}=i \omega_{n}+E_{D}+\cos ^{2} \alpha\left(n_{0}+n_{b}\right) t_{p}
\end{gathered}
$$

$$
\begin{gathered}
b_{12}=\cos \alpha R_{D}^{K}\left(n_{d}+n_{k}\right) t_{p} ; \\
b_{21}=\cos \alpha R_{D}^{K}\left(n_{0}+n_{b}\right) t_{p} ; \\
b_{32}=b_{14}=-c_{31}=c_{24}=-\cos ^{2} \alpha \Delta_{0}^{k} t_{p} ; \\
b_{23}=b_{41}=-c_{42}=-c_{13}=\left(R_{D}^{K}\right)^{2} \Delta_{0}^{k} t_{p} ; \\
b_{31}=-b_{43}=b_{24}=-b_{13}= \\
=c_{23}=-c_{14}=c_{41}=-c_{32}=\cos \alpha R_{D}^{K} \Delta_{0}^{k} t_{p} ; \\
b_{34}=b_{12}=c_{21}=c_{34}=\cos \alpha R_{D}^{K}\left(n_{d}+n_{k}\right) t_{p} ; \\
b_{43}=b_{21}=c_{12}=c_{43}=\cos \alpha R_{D}^{K}\left(n_{b}+n_{0}\right) t_{p} ; \\
c_{11}=i \omega_{n}-E_{B}-\cos ^{2} \alpha\left(n_{0}+n_{b}\right) t_{p} ; \\
c_{22}=i \omega_{n}-E_{K}+E_{D}-\left(R_{D}^{K}\right)^{2}\left(n_{d}+n_{k}\right) t_{p} ; \\
c_{33}=i \omega_{n}-E_{B}+E_{K}+\left(R_{D}^{K}\right)^{2}\left(n_{d}+n_{k}\right) t_{p} ; \\
c_{44}=i \omega_{n}+E_{D}+\cos ^{2} \alpha\left(n_{0}+n_{b}\right) t_{p} ;
\end{gathered}
$$

where $n_{b}, n_{0}, n_{d}$, and $n_{k}$ are the population densities of the energy states under investigation, which satisfy the condition

$$
n_{b}+n_{0}+n_{d}+n_{k}=1,
$$

$\omega_{n}=(2 n+1) \pi T ; \quad T$ is the absolute temperature; $n=0, \pm 1, \pm 2, \ldots$; and $t_{p}$ is the momentum representation of the transport integral $t_{i j}$.

In the case $E<0$ we obtained the same equations for the Green's function as (A4), in which we should make the exchange: $E_{B} \rightarrow E_{A}, E_{D} \rightarrow E_{C}$, and $R_{D}^{K} \rightarrow R_{C}^{K}$.

1. Yu. A. Izyumov, M. I. Katsnel'son, and Yu. N. Skryabin, Magnetism of Collectivized Electrons [in Russian ], Nauka, Moscow (1994).

2. J. J. Neumeier, Physica C233, 354 (1994).

3. J. S. Schilling and S. Klotz, Physical Properties of High Temperature Superconductors, vol. III. D. M. Ginsberg (ed.), World Scientific, Singapore (1992).

4. H. Takahashi and N. Mori, Studies of High Temperature Superconductors, vol. 16, Anant Narlikar (ed.), Nova Science Publishers, INC (1996).

5. J. Hubbard, Proc. Roy. Soc. A276, 238 (1963).

6. A. A. Kosov and V. E. Shilov, Fiz. Nizk. Temp. 22, 1032 (1996) [Low Temp. Phys. 22(9), 787 (1996)].

7. R. O. Zaitsev and V. A. Ivanov, Fiz. Tverd. Tela (Leningrad) 29, 2554 (1987) [Sov. Phys. Solid State 29, 1475 (1987)].

8. D. M. Newns, P. Pattnaik, M. Rasolt, and D. A. Papaconstantopoulos, Physica C153-155, 1287 (1988). 
9. P. B. Zyubin, V. A. Ivanov, and E. A. Ugolkova, Teor. and Matem. Fiz. 101, 304 (1994).

10. N. M. Plakida, V. Yu. Yushankhai, and I. V. Stasyuk, Physica C160, 80 (1989).

11. A. A. Abrikosov, L. P. Gor'kov, and I. E. Dzyaloshinsky, Methods of Quantum Field Theory in Statistical Physics, Prentice Hall, Englewood Cliffs, NJ (1963).

12. J. Schriffer, Superconductivity, Nauka, Moscow (1970).

13. M. B. Maple, L. M. Paulius, and J. J. Neumeier, Physica C195, 64 (1992).

14. J. J. Neumeier, M. B. Maple, and M. S. Torikachvili, Physica C156, 574 (1988).

15. Marsiglo and J. E. Hirsh, Phys. Rev. B41, 6435 (1990).

16. J. J. Neumeier, T. Bjoernholm, M. B. Maple, and Iv. K. Schuller, Phys. Rev. Lett. 63, 2516 (1992).
17. J. D. Jorgensen, S. Pei, D. Lightfoot, D. G. Hinks, B. W. Veal, B. Dabrowski, A. D. Daulikas, R. Kleb, and I. D. Brown, Physica C171, 93 (1990).

18. J. Yu and A. F. Freeman, Phys. Rev. Lett. 58, 1035 (1987).

19. N. Tanahashi, I. Ye, T. Tamegai, C. Murayama, N. Mori, S. Yomo, N. Okazaki, and K. Kitazawa, Jpn. J. Appl. Phys. Lett. 28, L762 (1989).

20. J. E. Schirber, E. L. Venturini, J. F. Kwak, D. S. Ginley, and B. Morosin, J. Mater. Res. 2, 421(1987).

21. H. Uwe, T. Osada, A. Iyo, K. Murata, and T. Sakudo, Physica C162-164, 743 (1989).

22. N. Mori, C. Murayama, H. Takahashi, H. Kaneko, K. Kawabata, I. Ye, S. Uchida, H. Takagi, M. Tokura, T. Kubo, H. Sasakura, and K. Yamaya, Physica C185-189, 743 (1991). 